\title{
Open Source in Web-based Periodicals
}

\author{
Andres Baravalle and Sarah Chambers \\ Department of Computer Science, University of Sheffield, UK. \\ email: \{andres,sarah\}@dcs.shef.ac.uk
}

\begin{abstract}
In this paper we aim to investigate the role of the media in the diffusion of Open Source, analysing three web-based periodicals from Italy, United Kingdom and USA. The influence of the media in our society is wide and we have to look to that direction if we want to seriously investigate the indepth causes of the different trends. Nevertheless, our results show a picture that may not be familiar to many researchers of the field.
\end{abstract}

\section{Introduction}

The starting point for our research was the COSPA project which is investing the use of Open Source (OS) and Open Data Standards in the public administrations in Europe. It quickly became apparent that there was a different perception of Open Source across the different project partners and that OS is more positively perceived in some countries than others.

Decisions to use OS depends on numerous factors, including technical, economical and socio-cultural, but the information that the persons have plays a fundamental role [Lippmann 1950]. A question that arose was: why does OS has such different levels of acceptance and success in different countries, and what are the factors that influence it? We thought that these differences in opinion may be linked to the way that OS had been reported in the media the these countries and this is what we set to to investigate further.

\section{Method}

For our research, we selected three web-based periodicals The Register (UK); Punto Informatico (Italy) and C-Net News.com (USA). The periodicals are amongst the most read IT periodicals in their respective countries. At present we do not aim to identify global patterns or rules but specifically look at UK, Italy and USA.

To harvest the information from the periodicals, we developed a set of parsers, that can be used though a web interface. Using the parser, we have been able to collect information about more than 13,000 articles on OS, from 1998 until December 2005.

The core analysis is based on almost 500 articles published in the periodicals during November and December 2005. We analysed these articles categorizing them by level of relevance to OS. This was carried out to select the number of articles that

Please use the following format when citing this chapter:

Baravalle, A., and Chambers, S., 2006, in IFIP International Federation for Information Processing, Volume 203, Open Source Systems, eds. Damiani, E., Fitzgerald, B., Scacchi, W., Scotto, M., Succi, G., (Boston: Springer), pp. 347-348 
were discussing issues related to OS, compared to the number of articles that had just were just referring to $O S$ in passing. Articles were also classified by the topic of the article, the categories included: software, community and business.

\section{Results and Discussion}

In all the periodicals $O S$ is well represented. There is not a overload of information, with articles on OS in a ocean of articles on proprietary software. Punto Informatico had a higher percentage of articles related to OS that were actually focused on OS, while both The Register and C-Net News.com had a higher number of OS articles on business aspects. Punto Informatico had a higher ratio of articles on OS compared with the number of articles including the keyword "Microsoft".

However, both The Register and C-Net News.com have been featuring more articles on OS migrations compared with Punto Informatico. Moreover, Punto Informatico has been always the last periodical to report on the migrations.

\section{Conclusions}

Our experience working on the COSPA project in the UK and the failure in finding suitable test location sites for migrations lead us to consider whether the press in the UK was biased against OS or whether there was a lack of coverage on the ongoing migrations, compared to other countries like Italy. The data of our sample period shows that this is not true, and a more in-depth analysis shows that it is neither true using a longer time frame.

However, according to our research, OS was covered as well in the UK and USA as it was in Italy. In fact, the Italy periodical contained a few less articles on OS migrations.

Future work might focus more on the topics that have been covered in the webbased periodicals and we plan to continue our research investigating different causes, to try to define more clearly which are the significant factors that influence the adoption of $O S$.

\section{References}

Lippmann, W. 1950. Public opinion. New York : Macmillan. 